\title{
Propiedades psicométricas de la Escala de Ansiedad y Depresión Hospitalaria (HADS) en una muestra de pacientes mexicanos con enfermedades respiratorias
}

\author{
Psychometric properties of the Hospital Anxiety \\ and Depression Scale (HADS) in a sample \\ of Mexican patients with respiratory diseases
}

\author{
Alma Vanessa Madrigal González ${ }^{1}$, María Guadalupe Pablo Díaz ${ }^{1}$, \\ Joel Flores Juárez ${ }^{2}$ Eryka Urdapilleta Herrera ${ }^{3}$, Gabriela Lara Rivas ${ }^{3}$ \\ y Óscar Galindo Vázquez ${ }^{4}$ \\ Universidad Nacional Autónoma de México ${ }^{1}$ \\ Universidad Latina ${ }^{2}$ \\ Instituto Nacional de Enfermedades Respiratorias ${ }^{3}$ \\ Instituto Nacional de Cancerología ${ }^{4}$
}

Autor para correspondencia: Óscar Galindo Vázquez,psigalindo@yahoo.com.mx.

\begin{abstract}
RESUMEN
Introducción: La ansiedad y la depresión son problemas de salud mental prevalentes en pacientes con enfermedades respiratorias, por lo que su detección de forma válida y confiable es necesaria para que dichos pacientes reciban una oportuna y adecuada atención psicosocial. Objetivo: Validar la Escala de Ansiedad y Depresión Hospitalaria (HADS) en una muestra de pacientes con enfermedades respiratorias. Método: Se empleó un diseño transversal, instrumental, con un muestreo no probabilístico y por disponibilidad. Se incluyeron 260 participantes diagnosticados con enfermedades respiratorias, con un rango de edad de 15 a 95 años, de los cuales $50.4 \%$ eran hombres y 49.6 \% mujeres, especificándose los criterios de inclusión. Resultados: La muestra se caracterizó mayormente por sufrir enfermedades infecciosas y con comorbilidades. El análisis factorial confirmatorio de la HADS identificó dos factores, corroborando la estructura original. Discusión: La HADS mostró ser un instrumento válido y confiable para su uso en la atención clínica e investigación dirigida a pacientes con enfermedades respiratorias, población que puede requerir atención psicosocial durante la atención de su salud física.
\end{abstract}

Palabras clave: Ansiedad; Depresión; Enfermedades respiratorias; México; Validación.

\footnotetext{
${ }^{1}$ Facultad de Psicología, Av. Universidad 3004, Col. Copilco-Universidad, Del. Coyoacán, 04510 Ciudad de México, México, correos electrónicos: vanessa.madrigal.27@gmail.comy mag_pabdi@hotmail.com.

${ }^{2}$ Escuela de Psicología, Campus Sur unila, Av. Pedro Henríquez Ureña 173, Col. Los Reyes, Del. Coyoacán, 04330 Ciudad de México, México, correo electrónico: signus_dark@hotmail.com.

${ }^{3}$ Programa de Apoyo a Pacientes y Familiares, Calz. de Tlalpan 4502, Col. Belisario Domínguez, Secc 16, Del. Tlalpan, 14080 Ciudad de México, México, correos electrónicos: erykau@yahoo.com.mx y agabilara@hotmail.com.

${ }^{4}$ Unidad de Investigación y Desarrollo de la Psicooncología, Servicio de Psicooncología, Av. San Fernando 22, Col. Belisario Domínguez, Secc 16, Del. Tlalpan, 14080 Ciudad de México, México, tel. (55)56-28-04-00, ext. 60259, correo electrónico: psigalindo@yahoo.com.mx.
} 


\begin{abstract}
Introduction: Anxiety and depression symptoms are prevalent in patients with respiratory diseases. Thus detecting such symptoms in a valid and reliable way is relevant for developing and implementing timely and adequate psychosocial care. Objective: To validate the Hospital Anxiety and Depression Scale (HADS) in a sample Mexican patients with respiratory diseases. Method: An instrumental, cross-sectional design, with non-probability sampling, was used due to participant availability. A total of 260 patients diagnosed with respiratory diseases, aged from 15 to 95 years participated; $50.4 \%$ men and $49.6 \%$ women. Results: The sample was characterized mainly by infectious diseases and comorbidities. Confirmatory factor analysis on HADS data yielded two factors, thus corroborating the original structure. Discussion: HADS proved to be a valid and reliable instrument for its use in clinical care and research aimed at patients with respiratory diseases provided that these patients tend to require psychosocial care throughout the duration of their condition.
\end{abstract}

Keywords: Anxiety; Depression; Respiratory diseases; Mexico; Validation.

Recibido: 06/04/2020

Aceptado: 08/06/2020

\section{INTRODUCCIÓN}

$\mathrm{D}$ e acuerdo con la Organización Mundial de la Salud (OMS) (2018), las enfermedades no transmisibles son la causa principal de mortalidad en el mundo y causan 41 millones de defunciones al año, de las cuales 3.9 millones son por enfermedades respiratorias. En México, este tipo de padecimientos tienen considerables efectos en la salud pública, tal es el caso de la influenza de 2009 y el COVID-19 en 2019.

Cientos de millones de personas sufren cada día las consecuencias de una enfermedad respiratoria crónica (ERC). Según estimaciones de la OMS (2004), 235 millones padecen asma, 64 millones enfermedad pulmonar obstructiva crónica (EPOC) y millones más rinitis alérgica, así como otras ERC que a menudo no llegan a diagnosticarse. En esta misma línea, y según el estudio sobre la carga mundial de morbilidad, la OMS (2017) en 2016 evaluó la prevalencia de EPOC en 251 millones de casos y en 2017 estimó 235 millones de casos de asma; cabe mencionar que estas cifras están consideradas a nivel mundial.

Los foros de las sociedades respiratorias internacionales indican que las enfermedades de tipo respiratorio imponen una inmensa carga sanitaria en todo el mundo, y que cinco enfermedades respiratorias (EPOC en tercer lugar; infecciones de las vías respiratorias inferiores en el cuarto; cáncer traqueal, bronquial y pulmonar en el sexto; tuberculosis en el duodécimo, y asma en el vigésimo octavo) figuran entre las causas más comunes de muerte en todo el mundo.

Se estima que 65 millones de personas padecen EPOC de moderada a grave, de los cuales fallecen aproximadamente tres millones cada año, lo que convierte a esta enfermedad en la tercera causa de muerte en todo el mundo. Hay que señalar que las infecciones agudas de las vías respiratorias bajas se encontraron entre las tres principales causas de muerte. Se estima que los problemas de salud respiratorios causan casi 4 millones de muertes al año (por ejemplo, las infecciones del tracto respiratorio causadas por la influenza matan de 250 mil a 500 mil personas). En 2015, 10.4 millones de personas desarrollaron tuberculosis, $\mathrm{y}$ aproximadamente 1.4 millones murieron a causa de esta infección (Foro de las Sociedades Respiratorias Internacionales, 2017).

En este grupo de pacientes, los síntomas de ansiedad y depresión se han asociado con varias patologías crónicas respiratorias (Gada, Khan, DeFina y Sherwood, 2014). La prevalencia descrita de los síntomas de ansiedad en pacientes con EPOC es del 35-40\% (Yohannes y Alexopoulos, 2014), y está asociada a déficits cognitivos (Roncero et al., 2016). Las dificultades para respirar generan síntomas de ansiedad, y cuando son persistentes pueden manifestarse no tan solo como ansiedad generalizada, sino incluso como trastornos de pánico (Pothirat et al., 2015).

Miravitlles et al. (2014) identificaron en 727 pacientes $34.5 \%$ de síntomas de ansiedad y $27.6 \%$ de depresión, hallando que la presencia de disnea 
persistente genera mayores niveles de ansiedad y de depresión si se le compara con una manifestación con escasos síntomas. También se ha encontrado en pacientes con EPOC prevalencias de depresión que oscilan entre 6 y 75\% (Pothirat et al., 2015). Por su parte, Llanos y Ponce (2019) identificaron en pacientes con tuberculosis $76 \%$ de depresión, ocupando el síndrome depresivo mayor $6 \%$ de la misma. Por su parte Lugo, Reynoso y Fernández (2014) hallaron en pacientes mexicanos con EPOC que $50 \%$ se encontraban de leve a severamente deprimidos y que $75 \%$ presentaban ansiedad de leve a severa.

Los problemas de salud pública respiratorios globales han afectado considerablemente a la población mexicana, como la influenza ocurrida en 2009 (Córdova et al., 2009) y el COVID-19 durante el año pasado. Duan y Zhu (2020) han reportado diversas dificultades en la cooperación entre los servicios de salud comunitarios y las instituciones de atención de salud mental; por ejemplo, se dificultaba la asignación de profesionales de salud apropiados a las personas afectadas por la epidemia, y después de la remisión de la infección viral los pacientes no eran transferidos oportunamente de un hospital a una institución comunitaria de servicios de salud para recibir tratamiento psicológico continuo.

Estas necesidades psicológicas y las variaciones de la prevalencias en pacientes con enfermedades respiratorias pueden deberse a lo heterogéneo de las poblaciones estudiadas y a la diversidad de instrumentos utilizados, ya que en algunos casos se valoraban los síntomas de depresión y en otros se restringían a la ocurrencia de cuadros depresivos establecidos (Roncero et al., 2016) y adicionalmente a un posible subdiagnóstico; como se puede ver, el carecer de equipos de salud mental puede impedir la identificación de tales sintomatologías.

Se han desarrollado diversos instrumentos para evaluar los síntomas de ansiedad y depresión en pacientes médicamente enfermos, siendo una de las más utilizadas la Escala de Ansiedad y Depresión Hospitalaria (HADS), desarrollada por Zigmond y Snaith (1983), la cual ha sido muy útil en los medios hospitalarios no psiquiátricos y en la atención primaria. Este instrumento considera dimensiones cognitivas y afectivas y omite los aspectos somáticos (insomnio, fatiga, pérdida de apetito), evitando así atribuirlos a la enfermedad. Se ha utilizado este instrumento para fines propios de la atención clínica y para identificar los efectos de intervenciones psicosociales en poblaciones con enfermedades agudas y crónicas.

La HADS se ha utilizado en México para fines clínicos y de investigación y se ha validado en pacientes obesos (López et al., 2002), mujeres con diversas condiciones de enfermedad (Villegas, 2004), pacientes con cáncer de mama (Ornelas, Tufiño y Sánchez-Sosa, 2011), con VIH (Nogueda, Pérez, Barrientos, Robles y Sierra, 2013), con cáncer (Galindo et al., 2015), cuidadores primarios informales de pacientes con cáncer (Galindo, Meneses, Herrera, Caballero y Aguilar, 2015), personas con trastorno de la conducta alimentaria (Barriguete, Pérez, De la Vega, Barriguete y Rojo, 2017) y pacientes con enfermedades cardiovasculares (Herrera, Galindo y Lerma, 2018). En tales estudios se han reportado coeficientes alfa de Cronbach de entre 0.78 y 0.90 , así como estructuras factoriales similares a las de la versión original.

Sin embargo, hace falta una versión validada para población mexicana con enfermedades respiratorias; por consiguiente, el objetivo del presente estudio, con diseño no experimental, transversal e instrumental, fue validar la Escala de Ansiedad y Depresión Hospitalaria (HADS) en una muestra con este tipo de pacientes.

\section{MÉTODO}

\section{Participantes}

Se utilizó una muestra no probabilística en una institución mexicana pública de tercer nivel de atención hospitalaria, constituida por 260 pacientes de ambos sexos. La tabla 1 muestra las características generales de dicha muestra. 
Tabla 1. Descripción de la muestra $(\mathrm{N}=260)$.

\begin{tabular}{|c|c|c|c|c|c|}
\hline Género & f & $\%$ & Lugar de residencia & f & $\%$ \\
\hline Masculino & 131 & 50.4 & Ciudad de México & 129 & 49.6 \\
\hline Femenino & 129 & 49.6 & Interior de la república & 131 & 50.4 \\
\hline \multicolumn{3}{|c|}{ Escolaridad } & \multicolumn{3}{|l|}{ Ocupación } \\
\hline Ninguna & 19 & 7.3 & Campesino(a) & 13 & 5.0 \\
\hline Primaria & 72 & 27.7 & Comerciante & 31 & 11.9 \\
\hline Secundaria & 70 & 26.9 & Desempleado(a) & 39 & 15.0 \\
\hline Preparatoria & 51 & 19.6 & Empleado(a) & 34 & 13.1 \\
\hline Licenciatura & 46 & 17.7 & Estudiante & 10 & 3.8 \\
\hline Posgrado & 2 & 0.8 & Hogar & 86 & 33.1 \\
\hline \multicolumn{3}{|c|}{ Estado civil } & Jubilado(a)/pensionado(a) & 3 & 1.2 \\
\hline Soltero(a) & 72 & 27.7 & Oficio & 40 & 15.4 \\
\hline Casado(a) & 106 & 40.8 & Actividad profesional & 4 & 1.5 \\
\hline Viudo(a) & 25 & 9.6 & \multicolumn{3}{|l|}{ Religión } \\
\hline Unión libre & 39 & 15.0 & Católica & 211 & 81.2 \\
\hline Divorciado(a) & 11 & 4.2 & Cristiana & 21 & 8.1 \\
\hline Separado & 7 & 2.7 & Testigo de Jehová & 5 & 1.9 \\
\hline \multicolumn{3}{|l|}{ Diagnóstico } & Otros & 6 & 2.3 \\
\hline Intersticiales & 18 & 6.9 & Ninguna & 17 & 6.5 \\
\hline Infecciosas & 103 & 39.6 & \multicolumn{3}{|c|}{ Tipo de comorbilidad } \\
\hline Cáncer & 20 & 7.7 & Obesidad & 22 & 8.5 \\
\hline Obstructivas & 56 & 21.5 & Diabetes e hipertensión & 42 & 16.2 \\
\hline Procedimientos & 32 & 12.3 & Diabetes & 25 & 9.6 \\
\hline Quirúrgicas & 15 & 5.8 & Hipertensión & 30 & 11.5 \\
\hline Otros & 16 & 6.1 & Otros & 35 & 13.5 \\
\hline \multicolumn{3}{|l|}{ Pronóstico } & \multicolumn{3}{|c|}{ Consumo de sustancias } \\
\hline Bueno & 19 & 7.3 & No & 109 & 41.5 \\
\hline Malo & 14 & 5.4 & Sí & 151 & 58.1 \\
\hline Regular & 6 & 2.3 & \multicolumn{3}{|l|}{ Tipo de sustancia } \\
\hline Reservado a evolución & 221 & 85.1 & Alcohol & 34 & 13.1 \\
\hline \multicolumn{3}{|c|}{ Comorbilidad } & Tabaco & 27 & 10.4 \\
\hline Sí & 154 & 59.3 & Alcohol y tabaco & 75 & 28.8 \\
\hline No & 106 & 40.8 & Alcohol, tabaco y cocaína & 3 & 1.2 \\
\hline & & & Alcohol, tabaco y marihuana & 4 & 1.6 \\
\hline & & & Otros & 10 & 4.0 \\
\hline
\end{tabular}

\section{Procedimiento}

La obtención de los participantes fue por disponibilidad durante las visitas a hospitalización en cualquiera de los servicios entre agosto y marzo. Cada uno de los participantes aceptó participar en el presente estudio mediante la lectura, comprensión y firma del formato de consentimiento informado de investigación.
Criterios de inclusión: 1) Ser pacientes hospitalizados en cualquiera de los servicios clínicos por diagnóstico médico confirmado de enfermedad respiratoria, 2) que hubiesen sido evaluados dentro de las primeras 24 horas del ingreso hospitalario, 3) que aceptaran ser valorados mediante la HADS, 4) que respondieran todos los reactivos del instrumento, 5) no haber sido diagnosticados o tra- 
tados en los últimos seis meses por algún trastorno psiquiátrico, 6) no estar en tratamiento farmacológico o psicológico.

Criterios de exclusión: 1) Tener complicaciones vasculares mayores o disnea que impidiera la evaluación, 2) que mostraran deficiencias cognitivas, así como problemas auditivos o visuales severos, 3) estar intubados, 4) que no tuviesen trastornos de aprendizaje, 5) que hubieran sufrido traqueotomías.

Criterio de eliminación: 1) Que durante el llenado del instrumento el paciente decidiera no seguir contestándolo.

\section{Instrumentos}

\section{Cedula de identificación}

Se diseñó y aplicó una cédula de identificación del participante que incluía datos sociodemográficos (edad, sexo, nivel de estudios, estado civil) y clínicos (diagnóstico, pronóstico, tratamiento médico, comorbilidad médica y antecedentes de consumo de sustancias psicoactivas).

Escala de Ansiedad y Depresión Hospitalaria (HADS) (Zigmond y Snaith, 1983).

La HADS (Hospitalized Anxiety and Depression Scale por sus siglas en inglés) es un instrumento autoaplicable que consta de catorce reactivos: siete diseñados para medir los síntomas de ansiedad y siete los de depresión. Cada reactivo tiene cuatro posibles respuestas, que se puntúan de 0 a 3 . La HADS se puede contestar en 5 o 6 minutos; los pacientes con enfermedades crónicas degenerativas no tienen problemas para los reactivos, y son de fácil aplicación para el equipo de salud (Bjelland, Dahl, Haug y Neckelmann, 2002). Las instrucciones, reactivos y opciones de respuesta son comprendidos adecuadamente por parte de los pacientes.

\section{Análisis estadísticos}

Se efectuó una auditoría de la base de datos, $10 \%$ de la muestra, para determinar la confiabilidad. La selección de la muestra se llevó a cabo mediante un sistema de aleatorización en el programa SPSS, versión 21.0. Se obtuvo una puntuación total de 99.76 de $100 \%$, lo que indica que los datos son confiables.

Se hizo un análisis descriptivo de cada reactivo para asegurar que no hubiera datos extremos y que se contara con datos en todas las casillas resultantes. Se generó una nueva variable con la suma de todos los reactivos y se identificaron los cuartiles extremos, con los cuales se corrió una prueba $t$ de Student para dos muestras independientes, en la que se buscó significancia en todos los reactivos, demostrando con ello que podían discriminar entre tener o no el constructo medido.

Luego se hizo un análisis cruzado con todos los reactivos para confirmar que las puntuaciones de los cuartiles extremos identificados corrieran en direcciones opuestas. Finalmente, se identificó la estructura factorial mediante un análisis exploratorio de componentes principales con rotación varimax, mientras que el confirmatorio y la consistencia interna se hicieron mediante el coeficiente alfa de Cronbach.

\section{Análisis factorial exploratorio}

Se realizó este análisis con rotación varimax. Los criterios utilizados fueron cargas factoriales iguales o mayores a .40, y por lo menos dos ítems en un solo factor. Finalmente, se determinó su consistencia interna mediante el coeficiente de alfa de Cronbach, adoptándose un nivel de significancia estadística de $p<.05$ (Gardner, 2003).

\section{Análisis factorial confirmatorio}

Se evaluó el modelo con el mejor ajuste mediante un análisis factorial confirmatorio empleando el método de máxima verosimilitud a partir de la observación de límites aceptables de los estimadores, parámetros estandarizados, así como de la no colinealidad en las variables medidas. Se estimaron los índices de ajuste global $\chi^{2}$ y la razón $\chi^{2} / \mathrm{gl}$, los índices de bondad de ajuste (GFI), los indicadores adicionales (AGFI, TLI), así como el índice de bondad de ajuste comparativo (CFI) y la raíz cuadrada media del error de aproximación (RMSEA) (Byrne, 2010; George y Mailery, 2001; Kline, 2005). 


\section{RESULTADOS}

\section{Análisis factorial exploratorio}

El análisis de confiabilidad de la HADS obtuvo un coeficiente alfa de Cronbach total de 0.852 . El valor de la prueba KMO (Káiser-Meyer-Olkin) fue de $0.864(p=0.001)$. Debido a que las correlaciones internas fueron de medias a bajas, el análisis factorial se hizo mediante rotación varimax, ajustándose a dos factores que comprenden los 14 reactivos originales con autovalores arriba de 1 , que en conjunto explicaron $44.84 \%$ de la varianza, con cargas factoriales de sus reactivos que van de 0.496 a 0.729 . En la Tabla 2 se muestran las cargas factoriales de cada uno de los reactivos finales.

Tabla 2. Análisis factorial exploratorio de la HADS.

\begin{tabular}{|c|c|c|c|c|}
\hline $\begin{array}{l}\text { Coeficiente alfa de Cronbach global }(\alpha)=0.852 \\
\text { Varianza explicada }=44.84 \%\end{array}$ & \multirow{2}{*}{\multicolumn{2}{|c|}{ Carga factorial }} & $\begin{array}{r}\text { Varianza } \\
\text { explicada }\end{array}$ & $\begin{array}{c}\text { Alfa de } \\
\text { Cronbach }\end{array}$ \\
\hline HADS-A: Subescala de ansiedad & & & \multirow{8}{*}{$34.69 \%$} & \multirow{8}{*}{$\alpha=0.795$} \\
\hline 1. Me siento tenso(a) o nervioso(a). & 0.729 & -.011 & & \\
\hline 3. Tengo una sensación de miedo, como si algo horrible fuera a suceder. & 0.719 & .155 & & \\
\hline 13. Presiento una sensación de miedo muy intenso de un momento a otro. & 0.653 & .277 & & \\
\hline 9. Tengo una sensación extraña, como de aleteo o vacío en el estómago. & 0.642 & .091 & & \\
\hline 5. Tengo mi mente llena de preocupaciones. & 0.613 & .363 & & \\
\hline 11. Me siento inquieto(a), como si no pudiera parar de moverme. & 0.559 & .300 & & \\
\hline 7. Puedo estar sentado(a) tranquilamente y sentirme relajado(a). & 0.496 & .313 & & \\
\hline \multicolumn{3}{|l|}{ HADS-D: Subescala de depresión } & \multirow{8}{*}{$10.15 \%$} & \multirow{8}{*}{$\alpha=0.776$} \\
\hline 14. Me divierto con un buen libro, la radio o un programa de televisión. & .087 & 0.670 & & \\
\hline 6. Me siento alegre. & .388 & 0.655 & & \\
\hline 12. Me siento con esperanzas respecto al futuro. & .019 & 0.635 & & \\
\hline 4. Puedo reírme y ver el lado positivo de las cosas. & .240 & 0.633 & & \\
\hline 2. Todavía disfruto con lo que me ha gustado hacer. & .231 & 0.596 & & \\
\hline 10. He perdido el deseo de estar bien arreglado(a) o presentado(a). & .155 & 0.574 & & \\
\hline 8. Siento como si yo cada día estuviera más lento. & .223 & 0.566 & & \\
\hline M & 11.49 & 9.28 & & \\
\hline D.E. & 3.902 & 3.039 & & \\
\hline Varianza del factor & 15.224 & 9.236 & & \\
\hline Correlación intraclase del factor & $.352 a$ & $.314 a$ & & \\
\hline Valor inferior & .300 & .264 & & \\
\hline Valor superior & .408 & .369 & & \\
\hline Valor $F$ & 4.797 & 4.202 & & \\
\hline Valor $p$ & $\leq 0.001$ & $\leq 0.001$ & & \\
\hline
\end{tabular}

\section{Análisis factorial confirmatorio}

La razón $\chi^{2} / \mathrm{gl}$ de ajuste global $(95.1, p=0.001)$ del modelo ajustado a esta población tuvo una bondad de ajuste (GFI) de 0.931 y comparativo (CFI) de 0.935 , así como un índice RMR de 0.052 (próximo a 0) y RMSEA de 0.056 , por lo que el modelo concuerda con la versión original. Las cargas factoriales y la varianza del error explicado por cada reactivo pueden observarse en la Figura 1. 
Figura 1. Modelo AFC HADS $(\mathrm{n}=260) \cdot \chi^{2}=52.261, \mathrm{CMIN} / \mathrm{gl}=2.021 p \leq 0.0001, \mathrm{CFI}=0.961$, TLI $=0.952$, AGFI $=0.851$, RMR $=0.052$, RMSEA $=0.078(0.035-0.151)$, Hoelter, $\mathrm{n}=260(p \leq 0.01)$.

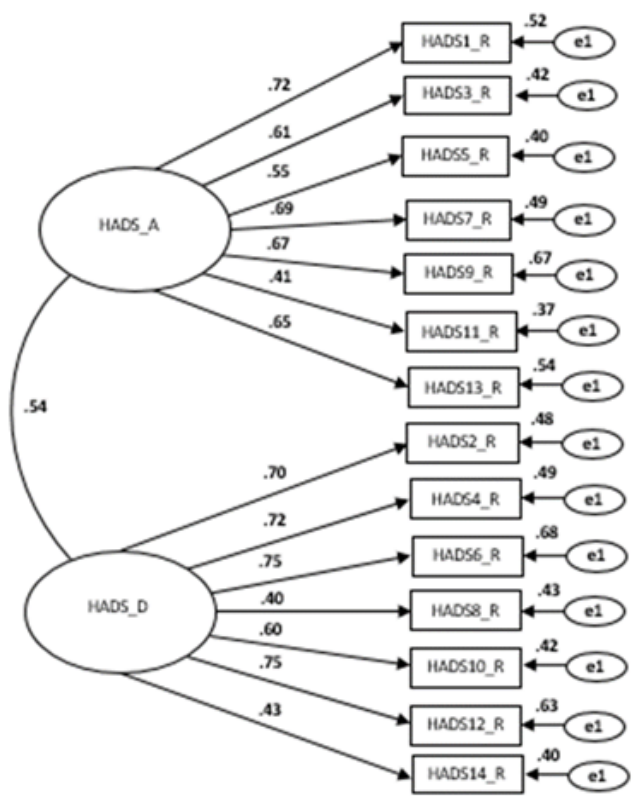

\section{Puntos de corte}

Se recodificó el sumatorio total y por factores dividiéndose por cuartiles $(25,50,75)$, con el propósito de obtener los puntos de corte y sus prevalencias. Los puntos de corte para los síntomas de ansiedad fueron los siguientes: de 0 a 8 , sin ansiedad o ansiedad mínima; de 9 y 10 , ansiedad leve; de 11 a 13, ansiedad moderada, y de 14 y más, ansiedad grave. En cuanto a los síntomas de depresión, los puntos de corte fueron, a saber: de 0 a 6 sin depresión o depresión mínima; de 7 y 8, depresión leve; de 9 y 10, depresión moderada, y de 11 y más, depresión grave. Los puntos de corte de la HADS total fueron: de 0 a 16 , sin síntomas o síntomas mínimos; de 17 a 19, síntomas leves; de 20 a 23, síntomas moderados, y de 24 en adelante, síntomas graves.

\section{DISCUSIÓN}

El presente estudio tuvo como propósito validar la HADS en una muestra de pacientes diagnosticados con enfermedades respiratorias, caracterizada por contar con el mayor porcentaje de enfermedades infecciosas (tuberculosis, influenza, neumonía), en su mayoría con un pronóstico reservado en su evolución, lo que implica la falta de certeza sobre su mejoría.

Se identificó una alta comorbilidad médica, siendo las enfermedades cardiometabólicas concomitantes las más prevalentes, con 59.3\%; adicionalmente, se identificó $58.1 \%$ de consumo de sustancias, entre las cuales las más frecuentes fueron el alcohol y el tabaco, lo que en conjunto dificulta una respuesta positiva a los esquemas terapéuticos y obstaculiza la mejoría de este grupo de pacientes durante y después del tratamiento (Cordoba et al., 2009). Lo anterior representa una población que requiere una atención multidisciplinaria extensa, de acuerdo a las múltiples necesidades de tratamiento médico y modificación de estilos de vida, en la cual la identificación de síntomas de ansiedad y depresión es primordial.

La HADS mostró una adecuada consistencia interna y una varianza explicada similares a las de otros estudios si se conserva la totalidad de los reactivos, lo que concuerda con la versión original de Zigmond y Snaith (1983), logrando así diferenciar dos factores de sintomatología psicológica ya detallada en estudios hechos con población mexicana con diferentes diagnósticos de enfermedades crónico-degenerativas, como pacientes con cáncer (Galindo et al., 2015; Ornelas et al., 2011), con 
trastorno de la conducta alimentaria (Barriguete et al., 2017) y en cuidadores primarios informales de pacientes con cáncer (Galindo et al., 2015). Sin embargo, de todas las validaciones hechas en México, solo el estudio de Luna et al. (2020) reportó el AFC en pacientes embarazadas, siendo compatible la estructura de dos componentes con la obtenida en la presente investigación, lo que confirma un modelo sencillo, parsimonioso y teóricamente relevante que corrobora el planteamiento original de la escala.

En relación a los puntos de corte, difieren de los establecidos en la versión original de Zigmond y Snaith (1983) y en otras poblaciones de pacientes mexicanos, como los señalados por Ornelas et al. (2011) en pacientes con cáncer de mama, y por Galindo et al. (2015) en pacientes con diferentes diagnósticos de cáncer, esto debido a la modificación del número de reactivos en cada subescala. Cabe señalar que la mayoría de los estudios hechos en México no los reporta (cf. Barriguete et al., 2017; Díaz, González, Sánchez y Fernández, 2018; Galindo et al., 2015; Herrera, Galindo y Lerma, 2018; López et al., 2002; Luna et al., 2020; Nogueda et al., 2013; Suárez et al., 2019; Villegas, 2004; Yamamoto et al., 2018).

La evaluación de los síntomas psicológicos de ansiedad y depresión presentes en los problemas respiratorios endémicos de México, o emergentes en el mundo por medio de la HADS representa una alternativa para un adecuado tamizaje en los servicios de salud mental; por ejemplo, en pandemias como la de la influenza en México de 2009 no fue un problema menor, lo que hace ver que los programas de salud mental específicos para las emergencias sanitarias son necesarios para abordar los efectos psicológicos en el personal sanitario, los pacientes, sus familias y la población en general (Cordoba et al., 2009).

Se recomienda, pues, utilizar esta versión con el objetivo de evaluar los efectos de las intervenciones psicosociales en quienes lo requieran, así como emplearla en las investigaciones relativas a la salud mental en este grupo de pacientes.

En conclusión, la HADS es un instrumento que mostró una estructura factorial de acuerdo a lo esperado, al igual que propiedades psicométricas adecuadas para la evaluación de la sintomatología de ansiedad y depresión en pacientes con enfermedades respiratorias. Esta versión concuerda en su estructura factorial con la original y con la utilizada en los otros estudios reportados previamente.

Se recomienda asimismo usarla en escenarios clínicos, especialmente con pacientes hospitalizados, como ocurrió en el presente estudio, y en la práctica ambulatoria, así como para evaluar los efectos de intervenciones psicológicas en pacientes con ERC con afectaciones psicosociales.

De la misma manera, su utilización en escenarios de tamizaje resulta sencilla y factible.

Entre las limitaciones de este trabajo se encuentra la falta de una entrevista clínica para confirmar los posibles casos de depresión o ansiedad que evidencien las variables a estudiar.

Se sugiere, por último, evaluar la estabilidad del instrumento a lo largo del tiempo, su sensibilidad al cambio de los procedimientos médicos, así como su efectividad para identificar los efectos de las intervenciones psicológicas en este grupo de pacientes.

Citación: Madrigal G., A.V., Pablo D., M.G., Flores J., J., Urdapilleta H., E., Lara R., G. y Galindo V., Ó. (2021). Propiedades psicométricas de la Escala de Ansiedad y Depresión Hospitalaria (HADS) en una muestra de pacientes mexicanos con enfermedades respiratorias. Psicología y Salud, 31(2), 323-332. https://doi.org/10.25009/pys.v31i2.2700.

\section{REFERENCIAS}

Barriguete, J., Pérez, R., De la Vega, R., Barriguete, P. y Rojo, L. (2017). Validación de la Escala Hospitalaria de Ansiedad y Depresión en población mexicana con trastorno de la conducta alimentaria. Revista Mexicana de Trastornos Alimentarios, $8(2), 123-130$.

Bjelland, I., Dahl, A., Haug, T. y Neckelmann, D. (2002). The validity of the Hospital Anxiety and Depression Scale. An updated literature review. Journal of Psychosomatic Research, 52(1), 69-77. 
Propiedades psicométricas de la Escala de Ansiedad y Depresión Hospitalaria (HADS) en una muestra de pacientes mexicanos...

Byrne, B. (2010). Structural equation modeling with AMOS: Basic concepts, applications, and programming. New York: Routledge.

Córdova V., J.A., Sarti, E., Arzoz P., J., Manuell L., G., Méndez J., R. y Kuri M., P. (2009). The influenza A (H1N1) epidemic in Mexico: Lessons learned. Health Research Policy \& Systems, 7(1), 21. Doi: 10.1186/1478-4505-7-21.

Díaz B., C.G., González C.R., A.L. M., Sánchez C., C.T. y Fernández V., M. (2018). Escala de Ansiedad y Depresión Hospitalaria (HADS) en mexicanos con asma: Confiabilidad y validez. Psicología Iberoamericana, 26(2), 75-87.

Duan, L. y Zhu, G. (2020). Psychological interventions for people affected by the COVID-19 epidemic. The Lancet Psychiatry, 7(4), 300-302.

Foro de las Sociedades Respiratorias Internacionales (2017). El impacto global de la enfermedad respiratoria (2a ed.). México: Asociación Latinoamericana de Tórax.

Gada, E., Khan, D., DeFina, L. y Sherwood, E. (2014). The relationship between asthma and self-reported anxiety in a predominantly healthy adult population. Annals of Allergy, Asthma \& Immunology, 112, 329-332.

Galindo, O., Benjet, C., Juárez, F., Rojas, E., Riveros, A., Aguilar, J., Álvarez, M. y Alvarado, S. (2015). Propiedades psicométricas de la Escala Hospitalaria de Ansiedad y Depresión (HADS) en una población de pacientes oncológicos mexicanos. Salud Mental, 38(4), 253-258.

Galindo, O., Meneses, A., Herrera, A., Caballero, M. y Aguilar, J. (2015). Escala Hospitalaria de Ansiedad y Depresión (HADS) en cuidadores primarios informales de pacientes con cáncer: propiedades psicométricas. Psicooncología, 12(2-3), 383-392.

Gardner, R. (2003). Estadística para psicología utilizando SPSS para Windows. México: Pearson Educación.

George, D. y Mailery, M. (2001). Using SPSS for Windows step by step: a simple guide and reference. Boston, MA: Aliyn \& Bacon.

Herrera, B., Galindo, O. y Lerma, A. (2018). Inventario de Ansiedad y Depresión Hospitalaria y Escala de Retiro de Patrón de Conducta tipo A en pacientes con enfermedades cardiovasculares: propiedades psicométricas. Psicología y Salud, 28(1).

Kline, R. (2005). Principles and practice of structural equation modeling. New York: Guilford.

Llanos T., F. y Ponce C., C. (2019). Depresión y adherencia en personas afectadas con tuberculosis. Una exploración preliminar de datos. Revista de Neuropsiquiatría, 82(2), 104-109. Doi: 10.20453/rnp.v82i2.3536.

López J., C., Vázquez, V., Arcilla, D., Sierra A., E., González, J. y Salín, R. (2002). Exactitud y utilidad diagnóstica del Hospital Anxiety and Depression Scale (HADS) en una muestra de sujetos obesos mexicanos. Revista de Investigación Clínica, 54(5), 403-409.

Lugo G., I.V., Reynoso E., L. y Fernández V., M. (2014). Percepción de enfermedad, depresión, ansiedad y control del asma: una primera aproximación. Neumología y Cirugía de Tórax, 73(2), 114-121.

Luna, D., Castañeda H., D.V., Guadarrama A., A.L., Figuerola E., R.P., García A., A., Ixtla P., M.B. y Lezama F., M.Á. (2020). Psychometric properties of the Hospital Anxiety and Depression Scale in Mexican pregnant women. Salud Mental, 43(3), 137-146.

Miravitlles, M., Molina, J., Quintano, J., Campuzano, A., Pérez, J. y Roncero, C. (2014). Factors associated with depression and severe depression in patients with COPD. Respiratory Medicine, 108, 1615-1625. Doi: 10.1016/j.rmed.2014.08.010.

Nogueda, M., Pérez, B., Barrientos, V., Robles, R. y Sierra, J. (2013). Escala de Ansiedad y Depresión Hospitalaria (HADS): validación en pacientes mexicanos con infección por VIH. Psicología Iberoamericana, 21(2), 29-37.

Organización Mundial de la Salud (2017). Enfermedad pulmonar obstructiva crónica (EPOC). Ginebra: OMS. Recuperado de http://www.who.int/es/news-room/fact-sheets/detail/chronic-obtuctive-pulmonary-disease-(copd).

Organización Mundial de la Salud (2018). Enfermedades no transmisibles. Ginebra: OMs. Recuperado de http://www.who.int/ es.news-room/fact-sheets/detail/noncomunicable-diseases.

Ornelas M., R., Tufiño, M. y Sánchez-Sosa, J. (2011). Ansiedad y depresión en mujeres con cáncer de mama en radioterapia: Prevalencia y factores asociados. Acta de Investigación Psicológica, 1(3), 401-414.

Pothirat, C., Chaiwong, W., Phetsuk, N., Pisalthanapuna, S., Chetsadaphan, N. e Inchai, J. (2015). Major affective disorders in chronic obstructive pulmonary disease compared with other chronic respiratory diseases. International Journal of Chronic Obstructive Pulmonary Disease, 10, 1583-1590.

Roncero, C., Campuzano, A., Quintan, J., Molina, J., Pérez, J. y Miravitlles, M. (2016). Cognitive status among patients with chronic obstructive pulmonary disease. International Journal of Chronic Obstructive Pulmonary Disease, 11, 543-551. Doi: 10.2147/COPD. S100850.

Suárez M., A., Petersen A., F., Almeida V., A., Robles G., R., Camacho, Á. y Fresán O., A. (2019). Psychometric evaluation of the Hospital Anxiety and Depression Scale in Mexican adults with ischemic and hypertensive cardiomyopathy. Archivos de Cardiologia de Mexico, 89(3), 221-226. Doi: 10.24875/ACME.M19000060.

Villegas, G. (2004). Reporte de experiencia profesional. Tesis inédita de maestría en Psicología. México: Universidad Nacional Autónoma de México. 
Yamamoto F., J.K., Sarmiento A., A., García A., M., Gómez G., L.E., Toledo M., J., Olivares G., L. y Fresán O., A. (2018). Escala de Ansiedad y Depresión Hospitalaria (HADS): Validación en pacientes mexicanos con enfermedad inflamatoria intestinal. Gastroenterología y Hepatología, 41(8), 477-482. Doi: 10.1016/j.gastrohep.2018.05.009.

Yohannes, A. y Alexopoulos, S. (2014). Depression and anxiety in patients with COPD. European Respiratory Review, 23, 345-349. Doi: 10.1183/09059180.00007813.

Zigmond, A. y Snaith, R. (1983). The Hospital Anxiety and Depression Scale. Acta Psychiatrica Scandinavica, 67, $361-370$. 